\title{
Molecular characterization of a proteolysis-resistant lipase from Bacillus pumilus SG2
}

\author{
R. Sangeetha ${ }^{1}$, I. Arulpandi ${ }^{2}$, A. Geetha ${ }^{3}$ \\ ${ }^{1}$ Department of Biochemistry, School of Life Sciences, Vels University, Chennai, India. \\ ${ }^{2}$ Research Department of Microbiology, Asan Memorial College, Chennai, India. \\ ${ }^{3}$ Department of Biochemistry, Bharathi Women's College, Chennai, India.
}

Submitted: October 1, 2012; Approved: March 14, 2014.

\begin{abstract}
Proteolysis-resistant lipases can be well exploited by industrial processes which employ both lipase and protease as biocatalysts. A proteolysis resistant lipase from Bacillus pumilus SG2 was isolated, purified and characterized earlier. The lipase was resistant to native and commercial proteases. In the present work, we have characterized the lip gene which encodes the proteolysis-resistant lipase from Bacillus pumilus SG2. The parameters and structural details of lipase were analysed. The lip gene consisted of $650 \mathrm{bp}$. The experimental molecular weight of SG2 lipase was nearly double that of its theoretical molecular weight, thus suggesting the existence of the functional lipase as a covalent dimer. The proteolytic cleavage sites of the lipase would have been made inaccessible by dimerisation, thus rendering the lipase resistant to protease.
\end{abstract}

Key words: Bacillus pumilus; covalent dimer; lipase; proteolytic-resistance.

\section{Introduction}

Proteases rank first among the three largest groups of industrial enzymes and account for about $60 \%$ of the total worldwide sale of enzymes. Lipases are the third largest group of enzymes and rank next to amylases and other carbohydrases (Sangeetha et al., 2011). Proteases and lipases have a wide range of applications and there are industrial processes which require a combination of a protease and a lipase. Effective breakdown of solids and the clearing and prevention of fat blockage or filming in waste systems are important in many industrial operations and they require a mixture of few enzymes like protease and lipase (Hsu et al., 2002). Similarly digestive aids, flavor enhancers, detergents and degumming agents contain both these enzymes (Olsen, 2000; O'Toole and Kun, 2003; Aehle, 2007; White et al., 2007). Therefore, concomitant production of both these enzymes will prove to be economical.

Proteases are hydrolytic enzymes which are capable of auto-digestion and also digest other enzymes which are produced simultaneously (Sangeetha et al., 2011). Thus concomitant production of both protease and lipase is feasi- ble only if the lipase is resistant to proteolytic degradation by the co-produced protease. A thorough study of literature reveals that the enhanced production of lipases may be contributed by one or more of the following factors like production parameters which do not promote protease production (Lopes et al., 2008), presence of protease inhibitors (Kok et al., 1996), or deletion of protease coding regions of the genome (Westers et al., 2005). Thus, protease plays a determinative role in the production of lipase.

Nevertheless, Henriette et al. (1993) and Ruchi et al. (2008) have reported the production of both these enzymes from Serratia marescens and Pseudomonas aeruginosa respectively. But the reason behind the proteolytic-resistant nature of the lipase was neither proposed nor deduced. We had earlier reported the production and characterization of a protease and a proteolysis-resistant lipase from a Bacillus pumilus SG2 (Sangeetha et al., 2010a). The SG2 lipase was resistant to native protease and this observation inspired the further investigation on SG2 lipase. Though there are reports that suggest the involvement of protease in the down regulation or enhancement of lipase production in Acinetobacter calcoaceticus and Lactobacillus plantarum,

Send correspondence to R. Sangeetha. Biomedical Instrumentation Science Laboratory, Department of Advanced Zoology and Biotechnology, Loyola College, 600034 Chennai, India. E- mail: sara_dna@yahoo.co.in. 
there are no such studies reported with Bacillus species (Sangeetha et al., 2010b). Hence, the gene sequences encoding lipase produced by B. pumilus SG2 and few other parameters were studied to analyse the plausible cause for the proteolytic-resistant nature of SG2 lipase. The present report thus deals with understanding the basis behind the proteolytic-resistance exhibited by SG2 lipase.

\section{Materials and Methods}

\section{Bacterial strain}

A most promising strain, B. pumilus SG2 which produced both protease and lipase was isolated from a food processing industrial effluent (Sangeetha et al., 2010a) and maintained on nutrient agar slants at $4{ }^{\circ} \mathrm{C}$. The bacterium was grown in Luria-Bertani broth at $37{ }^{\circ} \mathrm{C}$ for genomic DNA isolation.

\section{Extraction and purification of intracellular lipase}

B. pumilus $\mathrm{SG} 2$ was allowed to grow for $24 \mathrm{~h}$ in Luria-Bertani broth at $32{ }^{\circ} \mathrm{C}$. At the end of the incubation period, the cells were harvested by centrifugation at $10,000 \mathrm{~g}$, for $10 \mathrm{~min}$ at $4{ }^{\circ} \mathrm{C}$. The packed cells were suspended in normal saline and washed till the supernatant was a clear fluid. The cells were then suspended in $10 \mathrm{~mL}$ phosphate buffer ( $\mathrm{pH}$ 7.0). The cell suspension was shaken vigorously in the presence of glass beads at $300 \mathrm{rpm}$ for $10 \mathrm{~min}$. The contents were centrifuged and the supernatant was harvested as the source of intracellular (IC) lipase. The IC lipase was purified by ammonium sulphate fractionation, dialysis and gel permeation chromatography, as described earlier (Sangeetha et al., 2010a). The purified IC lipase was lyophilized for further study.

\section{DNA preparation and sequencing}

Genomic DNA was prepared by the CTAB method described previously by Tripathi and Rawal (1998). The lipase (lip) gene amplifications were performed using primers described by Bell et al. (2002). The primers used for lip gene amplification were forward - 5'- GAG TCG TAT AAG ATG AAT AAG GGG GAA and reverse - 5'TAA TTC GTA TTT TGT CCT CCG CCG TTC. The PCR conditions were as follows: one denaturation step at $94{ }^{\circ} \mathrm{C}$ for $45 \mathrm{~s}, 30$ cycles of annealing at $60^{\circ} \mathrm{C}$ for $45 \mathrm{~s}$, extension at $72^{\circ} \mathrm{C}$ for $90 \mathrm{~s}$ except for the final cycle for which the extension proceeded for $5 \mathrm{~min}$. The PCR products were purified using QIAQuick (Qiagen) spin column. The purified DNA was resolved electrophoretically on $1 \%(\mathrm{w} / \mathrm{v})$ agarose gel to determine the molecular weight of the amplified product. The nucleotide sequence of the lip gene was determined using DTCS quick start Dye terminator kit (Beckman Coulter) and submitted to GenBank sequence database under the accession number GQ398414.

\section{Sequence analysis}

The sequenced gene data were subjected to basic local alignment search tool (BLAST) analysis in NCBI website. The nucleotide blast was run using megablast (high similar sequence) program with maximum target of 50. Further, the comparative sequence data were obtained from GenBank, NCBI and the phylogenetic tree was constructed. The sequenced gene data were aligned manually in multiple alignment mode using Clustal-X Ver.2.0 aligning tool and taxonomic position was determined by phylogenetic analysis using MEGA (Molecular Evolutionary Genetic Analysis) Ver.4.0 (Tamura et al., 2007). The amino acid sequences of the enzymes were obtained using Expasy Translation tool. The parameters and structures of the proteins were studied using ProtParam, ProtScale and PredictProtein servers.

\section{Results and Discussion}

We had earlier purified a proteolysis resistant lipase from B. pumilus SG2 using ammonium sulphate fractionation and gel filtration chromatography. The enzyme which was purified to 12-fold was characterized and its molecular weight was determined (Sangeetha et al., 2010a). The basis for the proteolytic-resistance exhibited by SG2 lipase was analysed in this study.

The coding region of SG2 lipase (lip) was amplified and the size of the amplified product was $650 \mathrm{bp}$. There are few reports on proteolysis resistant lipases (Ruchi et al., 2008; Zhang et al., 2008), but the gene sequences that encode these lipases have not been documented. The lip gene of SG2 was amplified to analyse the presence of consensus sequences, motifs in the gene and to study its homology with other Bacillus lipases. The lip gene of SG2 exhibited identity of 99,97 and $96 \%$ respectively with lipase coding sequences of B. pumilus XJU-13 (EF434173), Bacillus sp B26 (AF232707) and B. licheniformis (EU414610), exhibiting a similarity of 99,97 and $96 \%$ respectively (Figure 1). The identity with other $B$. pumilus lipase coding sequences (AY474914, EU482152 and DQ339137) was as low as $87 \%$. The identity with the outgroup $B$. thermoleovorans was found to be $32 \%$.

Analysis using ORF finder revealed 6 ORFs, the major ORF comprising of $628 \mathrm{bp}$ which encoded a polypeptide of 209 amino acid residues. A putative RBS (ribosomal binding site) was found in the region upstream from the initiation codon at position 1 (Figure 2). The GC content of the gene was $44.5 \%$. The deduced amino acid sequences were compared with other lipases of the Bacillus species. The oxyanion region and the catalytic pentapeptide were highly conserved.

SignalP, version 3.0 predicted a cleavage site between A31 and A32 and a 31- amino acid signal sequence was assigned from the $\mathrm{NH}_{2}$ terminal of the $\mathrm{SG} 2$ lipase protein. The mature protein consisted of 177 amino acids. The 


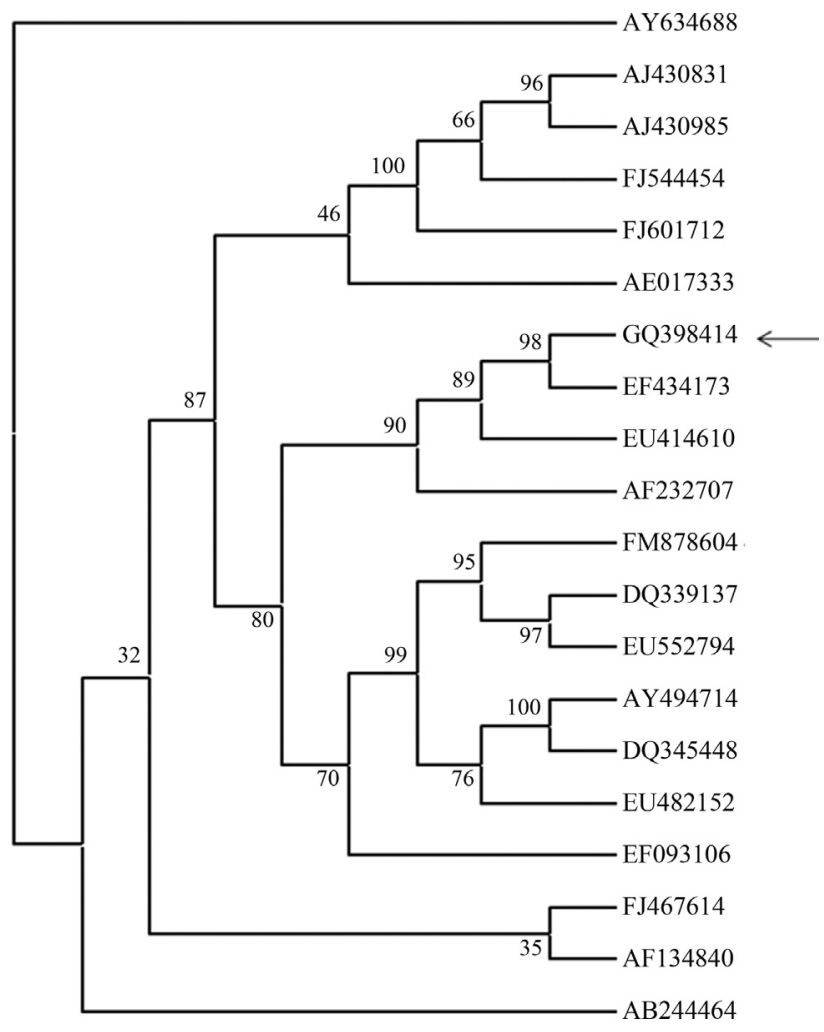

Figure 1 - Phylogenetic tree showing the genetic relationship between SG2 lipase and the other Family I lipases EF434173 - B. pumilus XJU-13 lipase; AF232707 - Bacillus sp. B26 lipase; EU414610 - B. licheniformis RSP - 09 lipase; EF093106 - B. pumilus F3 lipase precursor; AY494714 B. pumilus lipase precursor; EU482152 - B. pumilus B106 lipase (lipA); FM878604 - B. pumilus triacylglycerol lipase; DQ339137 - B. pumilus YZ02 lipase; DQ345448 - B. pumilus mutant lipase precursor; EU552794 - B. pumilus strain GMA1 lipase precursor; FJ544454 - B. subtilis lipase (lipW); AE017333 - B. licheniformis DSM13; FJ467614 - B. pumilus HZbp lipase; AB244464 - B. cereus C7-2; AF134840 - B. thermoleovorans lipase (ARA); AY634688 - B. coagulans strain 81-11 carboxylesterase; AJ430831 - B. megaterium lipA extracellular esterase; AJ430985 - Bacillus sp. BP-6 lipA extracellular esterase; FJ601712 - Synthetic construct clone JK lipM1 triacylglycerol lipase.

catalytic triad composed of Serine (S) 77, Aspartate (D) 133 and Histidine $(\mathrm{H}) 156$. The serine 77 residue appeared in the conserved pentapeptide sequence AHSMG (amino acids 75-79). The various Bacillus lipases known have, in common that an alanine residue replaces the first glycine in the conserved pentapeptide: Ala-Xaa-Ser-Xaa-Gly (Arpigny and Jaeger, 1999). SG2 lipase belongs to the $\alpha / \beta$-hydrolases superfamily and the enzymes belonging to this superfamily have the three amino acids of the catalytic triad in the order Ser-Asp-His. SG2 lipase belongs to Family 1 and subfamily 4 according to the classification proposed by Arpigny and Jaeger (Arpigny and Jaeger, 1999). Family 1 lipases are true lipases which demand special attention because their peculiar catalytic properties make them very attractive for industrial applications. Their marked preference for water-insoluble substrates and their adsorption on the oil/water interface before hydrolysis involve substantial

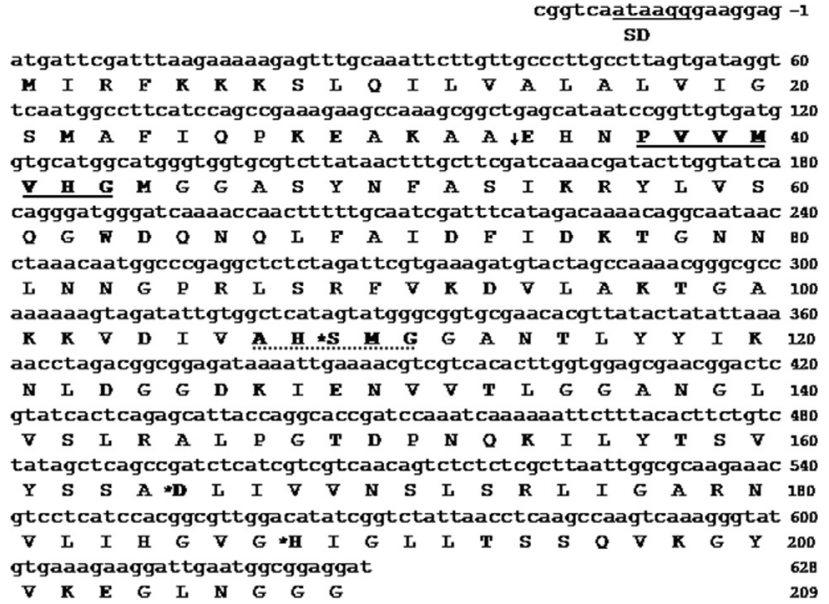

Figure 2 - Nucleotide and the deduced amino acid sequence of SG2 lipase. The Shine-Dalgarno (SD) sequence has been bolded and underlined. The oxyanion site conserved region has been underlined. The catalytic triad residues have been bolded with asterisk and the conserved sequence of $\mathrm{Ba}$ cillus lipases has been underlined with dotted line. The arrow indicates the cleavage site of the signal peptide.

conformational changes of the enzyme's architecture during catalysis (Arpigny and Jaeger, 1999).

PROF predictions revealed that the secondary structure of SG2 lipase contained $31.25 \%$ helices, $18.27 \%$ sheets and $50.48 \%$ loops. SG2 lipase was hence a mixed protein. About $52.4 \%$ of the amino acid residues of SG2 lipase had $16 \%$ of their surface exposed to solvents. Crystallographic and biochemical studies have shown that the mechanism of hydrolysis by lipases is similar to that of serine proteases. In both cases, an oxyanion created during hydrolysis is located in the so-called 'oxyanion hole' and is stabilized through interactions with some electrophiles (Kim et al., 1998). The sequences PVVMVHG present in the oxyanion-hole region of Bacillus lipases (Bell et al., 2002) was conserved in SG2 lipase at amino acid positions 5-11.

According to the lipase engineering database (LED), Bacillus lipases were clustered into groups 3,14 and 15. The lipases of group 14 were derived from three mesophilic Bacillus species, B. subtilis, B. pumilus and $B$. licheniformis that are known to secrete the smallest true lipases of approximately $20 \mathrm{kDa}$ and this group of lipases corresponds to "the homologous family Bacillus lipase" in LED (Kang et al., 2006). The calculated molecular weight of the mature form of SG2 lipase was $18.77 \mathrm{kDa}$. However, we had earlier determined its experimental molecular weight as $40 \mathrm{kDa}$. Lipase zymography revealed the presence of a band which corresponded to $40 \mathrm{kDa}$ molecular weight (Sangeetha et al., 2010a). Thus the experimental molecular weight was nearly double that of the calculated molecular weight. The PAGE and zymogram studies revealed the presence of a single lipase band (Sangeetha et al., 2010a). These observations suggest the existence of lipase in a dimeric form. Marginal differences between the 
theoretical and experimental values can be attributed to post-translational modifications of the enzyme. But, the experimental molecular weight of SG2 lipase was close to that expected for a dimer. Hence, SG2 lipase could have assumed a dimeric form after synthesis.

The absence of cysteine in the amino acid composition of the enzyme rules out the possibility of dimer formation mediated by a disulphide bond. Also the presence of a single protein band that corresponded to lipase in the SDSPAGE and zymogram studies (Sangeetha et al., 2010a) too confirm the absence of a disulphide or hydrogen bonding in the dimer. Thus, the lipase dimer would have formed by covalent bonding between the two monomers and the proteolysis-resistant nature of the SG2 lipase could be attributed to its presence in the functional dimer form. The dimerization of lipase may take place within the cell, prior to secretion or may be an extracellular dimerization event.

There are few reports on the existence of lipase dimers in bacterial and fungal species. Zhao et al. (2008) have reported a $65 \mathrm{kDa}$ dimeric lipase from $S$. marescens. Martinez and Schavez (2001) have purified a $58 \mathrm{kDa}$ lipase from $P$. aeruginosa. The lipase gene encoded a $30 \mathrm{kDa}$ enzyme and the dimer was formed due to post-translational modifications. The dimer did not denature into its monomers under SDS-PAGE conditions. The crystallographic analysis of the esterase of a thermophilic eubacterium, Alicyclobacillus acidocaldarius was performed which revealed an unexpected dimer. The dimer had formed as a consequence of a domain swapping involving its N-terminal region (De Simone et al., 2004). However, these studies have not analysed the proteolytic resistance of the dimeric lipases.

The $B$. pumilus proteases cleave at sites similar to chymotrypsin-like proteases (Han and Damodaran, 1998). The PeptideCutter tool of ExPASy Proteomics server showed 12 cleavage sites, with aromatic amino acids tyrosine $(\mathrm{Y})$ and phenylalanine $(\mathrm{F})$ at position 1 of the cleavage site in the SG2 lipase sequence. It could be presumed that the probable proteolytic cleavage sites in lipase were not exposed for attack by SG2 protease because of the existence of the functional lipase in its dimeric form.

\section{Conclusion}

The SG2 lipase was homologous to other Bacillus lipases. Thus the proteolysis-resistant nature of SG2 lipase was not due to its genetic make-up. Nevertheless, SG2 lipase in its functional form was found to be a dimer. Thus the proteolytic resistant nature of SG2 lipase could be attributed to its dimeric existence which perhaps would have masked its proteolytic cleavage sites making them inaccessible to native and commercial proteases.

\section{References}

Aehle W (2007) Industrial enzymes. In: Enzymes in Industry: Production and Applications. Culinary and Hospitality Industry Publication Services, Texas, pp 99-262.

Arpigny JL, Jaeger KE (1999) Bacterial lipolytic enzymes: Classification and properties. Biochem J 343:177-183.

Bell PJL, Sunna A, Gibbs MD, Curach NC, Nevalainen H.; Bergquist PL (2002) Prospecting for novel lipase genes using PCR. Microbiology 148:2283-2291.

De Simone G, Menchise V, Alterio V, Mandrich L, Rossi M, Manco G, Pedone C (2004) The crystal structure of an EST2 mutant unveils structural insights on the $\mathrm{H}$ group of the carboxylesterase/lipase family. J Mol Biol 343:137-146.

Han XQ, Damodaran S (1998) Purification and characterization of protease Q: A detergent- and urea-stable serine endopeptidase from Bacillus pumilus. J Agric Food Chem 46:3596-3603.

Henriette C, Zinebi S, Aumaitre MF, Petitdemange E, Petitdemange H (1993) Protease and lipase production by a strain of Serratia marcescens (532 S). J Ind Microbiol 12:129-135.

Hsu AF, Jones K, Foglia TA, Marmer WN (2002) Immobilized lipase catalysed production of alkyl esters of restaurant grease as biodiesel. Biotechnol Appl Biochem 36:181-186.

Kang HY, Kim JF, Kim MH, Park SH, Oh TK, Hur CG (2006) MELDB: A database for microbial esterases and lipases. FEBS Lett 580:2736-2740.

Kim HK, Park SY, Lee JK, Oh TK(1998) Gene cloning and characterisation of thermostable lipase from Bacillus stearothermophilus L1. Biosci Biotechnol Biochem 62:66-71.

Kok RG, Nudel CB, Gonzalez RH, Roodzant IMN, Hellingwerf KJ(1996) Physiological Factors Affecting Production of Extracellular Lipase (LipA) in Acinetobacter calcoaceticus BD413: Fatty Acid Repression of lipA Expression and Degradation of LipA. J Bacteriol 178:6025-6035.

Lopes M, Gomes N, Goncalves C, Coelho MAZ, Mota M, Belo I (2008) Yarrowia lipolytica lipase production enhanced by increased air pressure. Lett Appl Microbiol 46:255-260.

Martinez A, Chavez GS (2001) Characterization of the lipA gene encoding the major lipase from Pseudomonas aeruginosa strain IGB83. Appl Microbiol Biotechnol 56:731-735.

O'Toole DK, Kun LY, (2003) Enzyme modified food products. In: Kun, L.Y. (ed) Microbial Biotechnology: Principles and Applications. World Scientific Publishing Co. Pt. Ltd, Singapore, pp 307-320.

Olsen HS. 2000. Cleaning-in-place of soiled process equipment, esp, in dairy or slaughter house - by circulating a solution containing a protease and a lipase. U.S. Pat. 6071356.

Ruchi G, Anshu G, Khare SK (2008) Lipase from solvent tolerant Pseudomonas aeruginosa strain: Production optimization by response surface methodology and application. Bioresour Technol 99:4796-4802.

Sangeetha R, Geetha A, Arulpandi I (2010a) Concomitant production, partial purification and characterization of a serine protease and a proteolysis- resistant metallo lipase from $\mathrm{Ba}$ cillus pumilus SG2. Z Naturforsch C 65:61-65.

Sangeetha R, Geetha A, Arulpandi I (2010b) Concomitant production of protease and lipase by Bacillus licheniformis VSG1: Production, purification and characterization. Brazil J Microbiol 41:179-185. 
Sangeetha R, Arulpandi I, Geetha A (2011) Bacterial lipases as potential industrial biocatalysts: An overview. Res J Microbiol 6:1-24.

Tamura K, Dudley J, Nei M, Kumar S (2007) MEGA4: Molecular Evolutionary Genetics Analysis (MEGA) Software Version 4.0. Mol Biol Evol 24:1596-1599.

Tripathi G, Rawal SK (1998) Simple and efficient protocol for isolation of high molecular weight DNA from Streptomyces aureofaciens. Biotechnol Techniq 12:629-631.

Westers H, Braun PG, Westers L, Antelmann H, Hecker M, Jongbloed JDH, Yoshikawa H, Tanaka T, van Dijl JM, Quax WJ (2005) Genes Involved in SkfA Killing Factor Production Protect a Bacillus subtilis Lipase against Proteolysis. Appl Env Microbiol 71:1899-1908.
White DHG, Mair TS, Simpson JW, Taylor DJ, (2007) Drugs acting on the Gastro-intestinal system. In: Bishop, Y.M. (ed) The Veterinary Formulary. Pharmaceutical Press, London, pp 221-238.

Zhang Y, Menga K, Wang Y, Luo H, Yang P, Shi P, Wu N, Fan Y, Li J, Yao B (2008) A novel proteolysis-resistant lipase from keratinolytic Streptomyces fradiae var. k11. Enzyme Microb Technol 42:346-352.

Zhao LL, Xu JH, Zhao J, Wang ZL (2008) Biochemical properties and potential applications of an organic solvent-tolerant lipase isolated from Serratia marescens ECU1010. Process Biochem 43:626-633.

All the content of the journal, except where otherwise noted, is licensed under a Creative Commons License CC BY-NC. 